\title{
Revisiting video game ratings: Shift from content-centric to parent-centric approach
}

\author{
Hee Jhee Jiow ${ }^{1, *}$, Rayvinder Athwa ${ }^{1}$, Ling Ling Chew ${ }^{1}$, Muhammad Helmi Elias ${ }^{1}$, Nina \\ Lim $^{1}$, Kenneth Woo ${ }^{1}$ \\ ${ }^{1}$ Singapore Institute of Technology, 10 Dover Drive, Singapore 138683, Singapore
}

\begin{abstract}
The rapid adoption of video gaming among children has placed tremendous strain on parents' ability to manage their children's consumption. While parents refer online to video games ratings (VGR) information to support their mediation efforts, there are many difficulties associated with such practice. This paper explores the popular VGR sites, and highlights the inadequacies of VGRs to capture the parents' concerns, such as time displacement, social interactions, financial spending and various video game effects, beyond the widespread panics over content issues, that is subjective, ever-changing and irrelevant. As such, this paper argues for a shift from content-centric to a parent-centric approach in VGRs, that captures the evolving nature of video gaming, and support parents, the main users of VGRs, in their management of their young video gaming children. This paper proposes a Video Games Repository for Parents to represent that shift.
\end{abstract}

\section{Introduction}

The rapid adoption of video gaming among children has placed tremendous strain on parents' ability to manage their children's consumption [1]. To which parents rely on video games ratings (VGR) for information to support their mediation efforts [2]. Yet, there are many difficulties associated with such practice. This paper will highlight the challenges associated with current VGRs and argues for a shift from content-centric VGR approach to a parent-centric approach. This paper will begin by exploring several well renowned VGRs.

The Entertainment Software Rating Board (ESRB) lists a number of games and its ratings on their website, and even provides parental control guides to better manage their children's video gaming behavior [3]. Appropriate age warnings ranging from Early Childhood (EC) to Adults Only (AO) and brief content descriptors such as violence and sexuality were provided as well to better inform the users [3]. ESRB aims to provide information to empower parents in their video game management habits of their children.

The Pan European Game Information (PEGI) functions similarly to ESRB, with clear age restrictive warnings as shown from the numbers $3,7,12,16$ and 18 that corresponds with the respective age groups, and content labels in forms of pictures depicting violence (a

\footnotetext{
*Corresponding author: jhee.jiow@singaporetech.edu.sg
} 
picture of a fist) and foul language (a picture of various symbols in a speech bubble) [4]. PEGI advocates parental supervision and video game gate keeping, and provides assistance for consumer decision-making during video game purchase [4].

It is evident that VGRs generally provides video game content information to support parents' mediation efforts. However, there are two major issues with VGRs' content-centric approach - its subjectivity and its relevance.

\section{Subjectivity of Game Information}

First, this paper argues that such information is highly subjective considering how video games have evolved [1]. The validity of game ratings provided by the industry such as those from ESRB and PEGI have frequently been called into question, especially from parents $[5,6]$. Studies have shown that parents saw industry's game ratings to be too lenient and imprecise. While parents generally agree with industry when a video game is rated as unsuitable for children, those that were rated appropriate for children were mostly perceived differently by parents [6]. When parents were made to rate those games in comparison to ESRB's ratings, Walsh discovered that 13 percent of E-rated (for Everyone) video games were perceived as unsuitable for children aged 3 to 7 ; and 31 percent of Trated games were given $\mathrm{M}$ ratings by parents, highlighting the more conservative opinions of parents versus the industry [7]. Furthermore, Thompson \& Haninger's quantitative study of video games showed that more than half of $E$ rated games contained intentional violence, while less than half of those games were not issued with content descriptor [8]. Thompson, Tepichin \& Haninger found similar issues in their assessment of the accuracy of content descriptors in adult video games, where majority lacked content descriptors apart from violent content - thus questioning the validity and accuracy of many VGRs [5].

In addition, the exposure to the actual gameplay of some video games has caused parents to become more critical of industry's ratings, especially for violent content. A study by Becker-Olsen \& Norberg discovered that upon revealing actual gameplay sequences, parents change their perceived rating pre-exposure (based on game titles or box art) to a harsher rating [9]. Children, however were less sensitive and rated violent games more leniently. Such observation can be attributed to the fact that parents are more sensitive to video game violence, that has evolved to appear more realistic with the help of technological advancements. However, such discrepancy presents a risk of miscommunicating information by game industries to parents - where box art and game advertisements indicate game ratings that might be seen as 'misleading' for parents [9]. Furthermore, most parents use the television or movies rating system as a point of reference when providing ratings for games $[10,11]$, which also raises the issue of confusion in the systems across the different media platforms.

Differing opinions of game content between industry and parents is not the only factor that raises the subjectivity of VGR information. Additionally, the perception of what is suitable and unsuitable for different parents vary - in accordance with their norms, values and experience which shapes the expectations they have of their child [9]. Some parents were most concerned with violent content, while others found that foul language in video games was their biggest issue [10]. Other differences were also noted, such as fathers who have experiences in the army perceive war games as 'acceptable' violence for their child, whereas those involving elements of street violence were deemed unsuitable [10]. Hence, every parent has a different way of applying and interpreting VGRs, which raises perceptual bias and subjectivity of VGRs. In addition, different cultures reveal how parents might have varying perspective on what is deemed as appropriate. For example, parents in U.S. were more concerned with violent content in video games, whereas parents in more conservative places, such as Singapore, worry more about sexual themes $[12,13]$. 
While there are many calls for a universal rating system across all media types, scholars have argued against its feasibility because such approaches have overlooked some of the basic characteristics of video games as a medium - that they are impossible to be played in its entirety prior to the issuance of ratings $[9,14,15,16,7]$. Most importantly, video games have unique features such as "content variability, content modifiability and content meaning" [16, p.12] that differentiates itself beyond the static nature of other forms of media.

ESRB indicated how video games are "player-controlled and there are many different permutations of gameplay" [3, p.FAQ], which aptly describes content variability. Game content might change in accordance to how the user plays, hence players can choose from many different journeys in order to complete the various missions [16]. Free roaming games such as Grand Theft Auto (GTA), Bully and World of Warcraft (WoW) are examples of those that might provide multiple experiences in accordance to how the users decide to play them. In contrast, games such as Pac-Man and FIFA are more straightforward in terms of its content and the aim of the games, not allowing for much content variability. There are even games with no clear game narrative, such as Sims where players control the well-being of virtual families. Ultimately, the content experience of video games vary greatly which makes it difficult to convince consumers of a single rating or content indicator.

Moreover, gamers themselves contribute greatly to modification of the game content and gameplay experience [17]. ESRB specifies how "game experience may change during online play" [3, p.FAQ] and "online interactions not rated by the ESRB" [3, p.FAQ] on games that affords online engagements, in efforts to distance the industry from being responsible for how players interact in-games. Young gamers expose themselves to not just the content of the video game itself, but also the gaming 'culture' of others which might include the use of profanities in online voice chats $[18,19]$. In this situation, the actual game content does not contain strong language, but the experience of the user was altered by other players. Additionally, content modifiability can be experienced through "mods" $[16$, p.15] where programmers and other users construct their own variation of the game to provide a different experience. Moreover, these mods can be shared online for other users to play, thereby perpetuating the subjectivity of game content. In the life simulation game of Sims, the game developer originally censored the genitals of the avatars when they use the toilet or showers to keep it appropriate for the young. However, on an occasion, a usergenerated mod was made available online, which removed the censorship of the genitals and further created genitals for the avatars [16]. Another example would be the controversial mod known as Hot Coffee - an interactive sex scene in Grand Theft Auto: San Andreas that was rated M for Mature [11]. Although this particular content was not part of the original game, it demonstrates how modifications might greatly change the gameplay and experience of players, and thereby reduce the validity of game ratings. Unlike television programs and music, rating the content of video games by playing them in its entirety is impossible [20].

\section{Relevance of VGRs in the Evolving Video Game Space}

Second, this paper argues that content-centric approach adopted by VGRs is inadequate in this new media era. Jiow \& Lim described how the affordances of video games portability, sociability, perpetuity, accessibility, interactivity and identity multiplicity have evolved and raised concerns, and challenged parents' monitoring and management of their children's video gaming habits [1]. These concerns that parents have about video gaming revolve around other dimensions beyond content, namely time, interactions and effects [3, p.FAQ]. Parents are mainly concerned that prolonged video gaming could result 
in displacement of time intended for homework or studies [2, 21, 22, 23]. Parents are also concerned that their children will withdraw from healthy social activities as a result of spending excessive time on video gaming [22, 23]. Another concern is that children's academic grades will be compromised if they spend too much time playing video games [22]. Yet, some parents "sometimes used video games as a childcare management tool and reward, in hopes it would motivate the child to exhibit other positive behaviors" [2, p.123]. Parents are also concerned about the social interactions present within video gaming too. Concerns of social interactions goes beyond content modifications of the video game. Duggan \& Rainie noted that " $72 \%$ of parents of online teens are concerned about how their child interacts online with people they do not know, with some $53 \%$ of parents being 'very' concerned" [24, p.2]. Parents are typically worried that their child may be harassed, stalked, sexually exploited, cheated, mixed with the wrong company, or even subjected to unwanted advertising by online strangers $[2,25]$. The effects of video gaming on the children's social and academic functioning, which is also commonly used as a measure of pathological video gaming, is also a pressing concern among parents [26, 27, 2, 28]. Jeong \& Kim highlighted that excessive video gaming, particularly online gaming, has a strong correlation to the high absence of social relationships with family or peers - players indulge in the online world of social interaction while their social self-efficacy in reality are negatively affected [29]. They would spend more time fostering relationships in the online space and were reported to have more problematic offline relationships [29]. In addition, relationship problems with peers and teachers in school tend to result in poor grades as adolescents find it difficult to adapt to the school environment [29]. Hence, players use the online platform to escape from reality and into the virtual arena where they have that supposed 'freedom' to do anything. Internal issues such as loneliness, low self-confidence, anxiety and depression are some of the factors that may arise from excessive online video gaming and have the potential to affect the child's development both in the offline social arena as well as their academic aptitude. Parents are also concerned about the amount of money spent sustaining their children's video gaming habits - the effects of video gaming on finances - and typically frown upon video games that require payment [2].

\section{Shifting to a Parent-Centric Approach}

As such, a revisit of VGRs is required, and this study proposes an online VGR system, cogently named Video Games Repository for Parents (VGRP), that embodies a shift from content-centric approach to parent-centric approach. Doing so, would better support parents' efforts in mediating their children's video game use in the new media space. The next section describes and proposes that game duration, content, effect on players' occupational functioning and financial information be available in the VGRP.

As mentioned earlier, parents' main concern is about the displacement effect of time on video gaming children, specifically about effects of prolonged gaming, beyond the video gaming content [2]. VGRs generally do not provide data on the average amount of hours required to complete a particular game mainly because the amount of time required to play or complete it differs and varies from player to player, and game to game $[30,31,16]$. However, information highlighting the average time needed to complete a segment, quest or mission in the game would greatly aid parental mediation. Parents can then negotiate play time with their children either as a form of compromise or reward [2]. A major hurdle of parental concerns regarding time displacement is the lack of knowledge about new games. Traditional mediation efforts are largely based on a standard playing time given to their children however, these techniques operate on the assumption that all games operate on a standard unit of playtime. Yet, information regarding the average playtime of game is available from platforms that allow gamers to share their time taken to complete the game 
$[32,33]$. For example, games such as DOTA, where players are involved in skirmish battle mode, has each battle lasting for an hour on average, however for games such as WOW, players go on quests as long as 12 hours, as they progress further into the game [30, 34]. As such, this paper proposes that information on game duration be included in the VGRP since time is of paramount concern to parents, and such information is reasonably attainable.

While this paper argues a shift from content-centric approach, it does not call for an absence of game content information. Parents are concerned about the influences of game content on their children, especially violent, sexual or content that promotes values contrary to parents' beliefs. However, such game content information needs to be informative (allowing the user to judge for themselves) versus prescriptive (prescribing an age category to it). As such, this paper proposes that screenshots and/or gameplay sequences be shown in the VGRP.

Jiow found that parents were concerned about the effect of video game play on the occupational functioning of the children [2]. Studies have shown that prolonged video gaming has detrimental effects on the occupational functioning (mainly social and academic functioning) of young gamers [27, 35]. These effects may include "social withdrawal, self-neglect, family and marital problems as well as sleep deprivation and fatigue resulting in poor educational and/or work performance and job loss" [28, p.749]. While the presentation of this information may not be directly applicable to individual gamers (children), this paper proposes that Pathological Video Game Use data on specific games be included in VGRP.

It is paramount to note that whilst game content and duration information are causes of concern for parents, the financial cost of video games cannot be ignored. The financial cost of purchasing video games has always been a pertinent issue in the past, but then, it was restricted to the one-time purchase of the physical video games [36]. However, video games can now be played across various mediums and devices. And avails more opportunities for spending money without parental involvement. The financial concerns are more prevalent in the modern gaming industry as a variety of business tactics are used to encourage gamers to spend money on these video games. The evolution of business practices adopted by the gaming industry create a new demand for items that are deemed to be important in enhancing the gaming experience of players.

\section{Limitations and Conclusion}

This paper has explored the popular VGR sites, and highlighted the inadequacies of VGRs to capture the parents' concerns and to represent the evolving nature of video gameplay culture and consumption. This paper has argued for a shift from content-centric approach to a parent-centric approach in VGRs, and has also proposed an online VGRP to represent that shift. However, this paper based its analysis on existing literature, and has no concrete data on how parents might receive the proposed VGRP. As such, future studies may seek to qualitatively and quantitatively explore video game information that is desirable for parents to aid in their mediation efforts. The authors seek to do just that.

\section{References}

1. H.J. Jiow \& S.S. Lim, B. Sci. Technol. Soc. 32, 455 (2012)

2. H.J. Jiow, Parental mediation of video games in Singapore (Doctoral dissertation, National University of Singapore, Singapore 2014)

3. Electronics Software Rating Board, http://www.esrb.org/ratings/ratings guide.aspx (2016) 
4. Pan European Game Information, http://www.pegi.info/en/index/id/33/ (2016)

5. K.M. Thompson, K. Tepichin, K. Haninger, Arch. Pediat. Adol. Med. 160, 402 (2006)

6. D.A. Walsh, D.A. Gentile, Pediatrics. 107, 1302 (2001)

7. D.A. Walsh, Sixth annual video and computer game report card (Report 2001)

8. K.M. Thompson, K. Haninger, JAMA-J. Am. Med. Assoc. 286, 591 (2001)

9. K.L. Becker-Olsen, P.A. Norberg, J. Advertising. 39, 83 (2010)

10. A.D. Brekke, Video game ratings: Does the system work for parents? (Master's thesis, University of Tennessee, Knoxville 2006)

11. A. Brekke, C. Lepre, Burned by Hot Coffee?: Parental trust and usage of the video game rating system (International Communication Association 2007)

12. M.K. Miller, Polit. Pol. 38, 705 (2010)

13. P. Nikken, J. Jansz, S. Schouwstra, Eur. J. Commun. 22, 315 (2007)

14. A. Lenhart, J. Kahne, E. Middaugh, A. Rankin Macgill, C. Evans, J. Vitak, Teens, Video Games, and Civics: Teen's gaming experiences are diverse and include significant social interaction and civic engagement: Prepared by Pew Research Center $\&$ the MacArthur Foundation (Report 2008)

15. N.J. Stroud, A. Chernin, J. Child. Media. 2, 1 (2008)

16. J. Tocci, Rated M for Moral Panic: Content considerations in video game legislation (National Communication Association 2007)

17. M.D. Griffiths, Educ. Health. 28, 65 (2010)

18. H.J. Jiow, S.S. Lim, J. Lin, Game On: Refining the parental mediation framework for a video gaming landscape (International Communication Association 2016)

19. S. Livingstone, E.J. Helsper, J. Broadcast. Electron. 52, 581 (2008)

20. J.B. Funk, G. Flores, D.D. Bunchman, J.N. Germann, Youth. Soc. 30, 283 (1999)

21. A. Khoo, Asia. Pac. J. Educ. 32, 416 (2012)

22. L.A. Kutner, C.K. Olson, D.E. Warner, S.M. Hertzog, J. Adolescent. Res. 23, 76 (2008)

23. M.M. Skoric, L.L.C. Teo, R.L. Neo, Cyberpyschol. Behav. 12, 567 (2009)

24. M. Duggan, L. Rainie, Cell Phone Activities 2012: Prepared by Pew Research Center $\&$ the MacArthur Foundation (Report 2012)

25. A. Lenhart, O. Lewis, L. Rainie, Teenage Life Online: Prepared by Pew Research Center \& the MacArthur Foundation (Report 2001)

26. D.A. Gentile, Psychol. Sci. 20, 594 (2009)

27. D.A. Gentile, H. Choo, A. Liau, T. Sim, D. Li, D. Fung, A. Khoo, Pediatrics. 127, 319 (2011)

28. T. Sim, D. Gentile, F. Bricolo, G. Serpelloni, F. Gulamoydeen, Int. J. Ment. Health. Addict. 10, 748 (2012)

29. E.J. Jeong, D.H. Kim, Cyberpsychol. Behav. Soc. Netw. 14, 213 (2011)

30. J. Billieux, M. Van Der Linden, S. Achab, Y. Khazaal, L. Paraskevopoulos, D. Zullino, G. Thorens, Comput. Human. Behav. 29, 103 (2013)

31. Interactive Software Federation of Europe, Videogames in Europe: Consumer Study (Report 2012)

32. IGN, http://ap.ign.com/ (2016)

33. AskAboutGames, http://www.askaboutgames.com/ (2016)

34. F. Karlsen, Int. J. Game. Stud. 8 (2008)

35. D.A. Gentile, C.A. Anderson, Handbook of Children, Culture and Violence (Sage Publication 2010)

36. P. Zackariasson, T.L. Wilson, Com. For. 11, 56 (2013) 